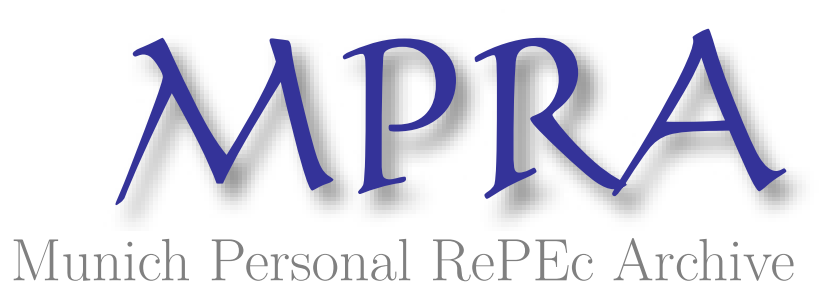

\title{
Aspects of Stickiness in Understanding Inflation
}

Kim, Minseong

30 April 2016

Online at https://mpra.ub.uni-muenchen.de/71036/

MPRA Paper No. 71036, posted 02 May 2016 13:39 UTC 


\title{
Aspects of stickiness in understanding inflation
}

\author{
Minseong Kim
}

$2016 / 04 / 30$

\begin{abstract}
This paper examines the question of how conventional understanding of inflation relates to stickiness. Several results that often go unnoticed are re-examined.
\end{abstract}

\section{Examining equations}

Suppose that economy has constant natural real interest rate $r_{t, n}=r$ all the time. Let Taylor rule be,

$$
i_{t}=r+\phi_{\pi} \pi_{t}+\phi_{y} \tilde{y}_{t}
$$

where $\tilde{y}_{t}$ is output gap, $\pi_{t}$ is inflation rate.

By the Fisher equation,

$$
i_{t}=r+E_{t}\left[\pi_{t+1}\right]
$$

Combining two and assuming $\phi_{y}=0$, as in [1],

$$
E_{t}\left[\pi_{t+1}\right]=\phi_{\pi} \pi_{t}
$$

If equilibrium real variables are unaffected by $\pi_{t}$ alone, then one may say that while real explosions are ruled out by some conditions, nominal explosions cannot be ruled out and thus Equation 3 allows explosive solutions. Unless $\pi_{t}=0$, any expected path explodes if $\left|\phi_{\pi}\right|>1$, as [1] says.

Now introduce New Keynesian Phillips Curve (NKPC), which assumes some degree of price stickiness:

$$
\pi_{t}=\beta E_{t}\left[\pi_{t+1}\right]+\kappa \tilde{y}_{t}
$$

Substituting the equation into Equation 3,

$$
\tilde{y}_{t}=\frac{\left(1-\beta \phi_{\pi}\right) \pi_{t}}{\kappa}
$$

- If $\beta \phi_{\pi}=1$, then $\tilde{y}_{t}=0$ at every $t$. Ruling out real variable explosions does not rule out nominal explosions. 
- If $\left|\phi_{\pi}\right|>1$ but $\beta \phi_{\pi} \neq 1$, then either $\pi_{t}=0$ at every $t$ which ensures $\tilde{y}_{t}=0$ at every $t$, or $\tilde{y}_{t}$ explodes. If real variable explosions are to be always ruled out, then $\left|\phi_{\pi}\right|>1$ ensures $\pi_{t}=0, \tilde{y}_{t}=0$.

- If $\left|\phi_{\pi}\right|<1$, then real variable explosions are avoided always. But nominal variable explosions cannot be ruled out by ruling out real variable explosions.

Thus, this gives us another type of divine coincidence - if $0<\beta<1$ and assuming that real explosions are eliminated automatically, then just setting $\left|\phi_{\pi}\right|>1$ eliminates sticky price distortions. While NKPC is only an approximation, its validity stands, as it is approximated around zero inflation steady state. Monetary policy is basically invisible. Thus, there is no need in this theoretical economy to adjust interest rate based on output gap and doing so only complicates the matter by creating an unnecessary business cycle.

The problem now is this: as demonstrated above, now the theory of inflation relies on existence of some type of stickiness that allows one to affect real variables by nominal variable changes. Here, $\tilde{y}_{t}$ is affected by $\pi_{t}$. Thus, ruling out explosion of $\tilde{y}_{t}$ allows one to rule out explosion of $\pi_{t}$.

But in an economy that does not allow real variables to be affected by nominal variable changes, such an understanding cannot be used to rule out explosion of $\pi_{t}$.

This now brings the question of faith: one may believe that there must be one rule/unified interpretation that allows how inflation rate or price level is determined for both flexible/frictionless and price-sticky or any sticky economies. Some others may believe that different rules can apply for frictionless and flexible economies. I will examine the former only.

For the former belief, one possible stance may be that "Standard New Keynesian theory of inflation is invalid." This stance would argue that there is no way to reconcile the equations into providing unique inflation path for a flexible economy. The following classical logic argument follows independent of the stance.

banning real explosions is valid $\rightarrow$ NK understanding of inflation is valid

By "banning real explosions is valid," it is meant that selection of a unique equilibrium by eliminating real-explosive equilibria is always valid, provided the model is consistent. Then assuming the model is true, "NK understanding of inflation is valid."

The aforementioned stance states that this understanding is invalid even if the model is true. Thus, a believer in this stance must be ready to say that obtaining a unique equilibrium by banning real explosions may not always be a valid way.

\subsection{Can real explosion elimination be justified?}

In any case, New Keynesian models need real explosion elimination to obtain a unique equilibrium. But why would an agent wish to have non-explosive 
equilibrium? The reason may be found on utility based on consumption-leisure tradeoff. Since actual labor quantity equilibrium depends on the specification of supply side, one cannot generalize completely, but it is in general safe to say that keeping magnitude of output gap under a certain bound is a welfare-wise good idea. The question then is whether the agents have power to enforce the hope as part of equilibrium selection. The households in general are constrained by the supply side too, so it is natural to say that there must be expectation that central bank can control output gap and inflation. But there is nothing in the model that justifies such an expectation.

\subsection{Is $\left|\phi_{\pi}\right|>1$ a threat to explode the economy if a target equilibrium is not met?}

Cochrane in [1] argues that setting $\left|\phi_{\pi}\right|>1$ for $E_{t}\left[\pi_{t+1}\right]=\phi_{\pi} \pi_{t}$ amounts to central bank ensuring nominal uniqueness by promising to explode the nominal economy if $\pi_{t}$ does not turn out to be the unique inflation rate obtained after elimination procedures. But as Cochrane says,

$$
\pi_{t+1}=\phi_{\pi} \pi_{t}+\delta_{t+1}
$$

where $\delta_{t+1}$ can be any arbitrary random variable with $E_{t} \delta_{t+1}=0$. In many rational expectation models, an agent is forward-looking - thus, it is $E_{t} \pi_{t+1}$ that affects $\pi_{t}$, not $\pi_{t}$ affecting $E_{t} \pi_{t+1}$. After $\pi_{t}$ is decided, it is mainly sunspot $\delta_{t+1}$ that decides $\pi_{t+1}$. This sunspot reflects the fact that expectation of future inflation is not anchored. Thus, even if $\left|\phi_{\pi}\right|>1$, there is no reason to see why the economy would necessarily go explosive if the target equilibrium is not met. The fact $\pi_{t}$ is not the target inflation rate reflects the fact that the household expectation for $\pi_{t+1}$ was different from the central bank's forecast. If this gap can be eliminated, then even if the economy started out with the gap, the nominal economy will not explode.

Thus, the actual problem again is whether central bank can make the agents believe that it has control over $y_{t}$ and $\pi_{t}$.

\section{Conclusion}

It is also known that if price level has floor and ceiling, then explosive solutions can be eliminated. Thus, all the aforementioned discussions do rely on the space of price level not being constrained. One can, as Cochrane in [1] argues, justify the space of price level not being constrained by hyperinflation episodes in history, but whether this is justifiable remains outside the scope of this paper. To summarize, what matters in the end is whether central bank and agents can agree on expected future inflation rate and output gap. As learning mechanism is missing from the model, one is still left with possibility of exploding real and nominal equilibria. And if justification of ruling out explosive real equilibria is made, then at least in simple New Keynesian models, one loses the reason to 
set $\phi_{y}$ other than zero, since $\left|\phi_{\pi}\right|>1$ fixes inflation rate to zero and output gap to zero.

\section{References}

[1] Cochrane, J. (2011). "Determinacy and Identification with Taylor Rules", Journal of Political Economy 119 (3): 565-615. 